November 18, 2018

DFTT $18 / 2001$

FNT-T 16/2001

SISSA $56 / 2001 / \mathrm{EP}$

hep-th/0107112

\title{
Implementing holographic projections in Ponzano-Regge gravity
}

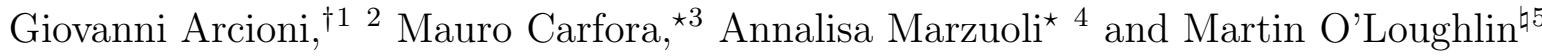 \\ † Dipartimento di Fisica Teorica, Università di Torino, \\ INFN, Sezione di Torino, \\ Via P. Giuria 1, I-10125 Torino, Italy \\ * Dipartimento di Fisica Nucleare e Teorica, Università degli Studi di Pavia, \\ INFN, Sezione di Pavia, \\ Via Bassi, 6, I-27100 Pavia, Italy \\ ${ }^{\natural}$ S.I.S.S.A. Scuola Internazionale Superiore di Studi Avanzati, \\ Via Beirut 4, I-34014 Trieste, Italy
}

\begin{abstract}
We consider the path-sum of Ponzano-Regge with additional boundary contributions in the context of the holographic principle of Quantum Gravity. We calculate an holographic projection in which the bulk partition function goes to a semi-classical limit while the boundary state functional remains quantum-mechanical. The properties of the resulting boundary theory are discussed.
\end{abstract}

PACS 04.60 Nc; $11.10 \mathrm{Kk}$

Keywords: discretized holography, Ponzano-Regge model, lattice gauge theories.

\footnotetext{
${ }^{1}$ Address after October 2001: Spinoza Institute, Utrecht, The Netherlands

2 E-mail: arcioni@to.infn.it

${ }^{3}$ E-mail: mauro.carfora@pv.infn.it

${ }^{4}$ E-mail: annalisa.marzuoli@pv.infn.it

${ }^{5}$ E-mail: loughlin@sissa.it
} 


\section{Introduction}

According to the holographic principle [1, 2] Quantum Gravity with some prescribed asymptotic behavior can be described by a theory defined on the boundary at infinity. Two recent examples which display holographic behavior are Matrix Theory [3] and the AdS/CFT correspondence [4. In particular regimes and by considering suitable decoupling limits it has been possible to carry out many checks of such a behavior and they all supported the holographic nature of theories containing gravity (for an exhaustive review see [5]). This represents a new perspective which seems to indicate that spacetime physics emerges from an underlying theory living in lower dimensions.

The holographic principle has been originally proposed by 't Hooft [1] in discussing spacetimes containing black holes. In particular, the fact that the black hole entropy is proportional to an area supports the holographic principle and the black hole horizon is then interpreted as a screen encoding bulk information. This lead 't Hooft to fomulate an S-matrix Ansatz program so as to properly incorporate black holes when dealing with quantized gravity. Since black holes carry the strongest possible gravitational field in any given volume they form a very natural upper bound of the energy spectrum.

A very interesting feature of the 't Hooft picture is that the theory living on the horizonscreen of the black hole is supposed to be some sort of discretized model [6] which comes about when including the transverse gravitational force in the construction of the Smatrix. According to preliminary attempts to implement such a picture, the operators on the discretized horizon satisfy an angular momentum algebra and the horizon is divided into various domains representing the various representations of the algebra. In the $2+1$ dimensional case [0] the horizon is described as being built up from string bits with unit lengths and a covariant algebra of observables associated to ingoing and outgoing radiation can be indeed constructed.

In such a framework it seems particularly interesting to examine discretized models in the presence of a boundary as a possible test of the holographic description of spacetime. One of the simplest and most natural scenarios is to consider the Ponzano-Regge model in three dimensions in the presence of a boundary. For a general introduction to discretized gravity and to the Ponzano-Regge model in particular see the review [8]. We will work in the Euclidean sector and as a preliminary investigation we examine the possibility of implementing a holographic description in the presence of a boundary. More precisely, we will try to implement some sort of holographic projection of bulk data precisely in the spirit of 't Hooft ideas.

In this connection it is worthwhile stressing that the continuum counterpart of the PonzanoRegge model is three-dimensional Chern-Simons theory [9] and it is well known that for gauge group $S L(2, R) \times S L(2, R)$ this latter induces on the boundary a WZW model and eventually Liouville field theory. This sort of "intrinsic" holographic behaviour has also led to the conjecture that a suitable higher dimensional Chern-Simons theory with some prescribed symmetries can be a possible candidate for a holographic description of M-theory [10]. Chern-Simons theory has indeed a very big symmetry group resembling gravity diffeomorphism invariance and this should lead to a holographic behavior when putting a suitably conditioned boundary in the theory. In view of these remarks it seems therefore interesting to analyze the presence of a boundary in a discretized version of Chern-Simons theory starting precisely from the three dimensional case. 
The paper is organized as follows: in Section 2 we briefly recall some aspects of the Ponzano-Regge model in the presence of boundary. In Section 3 we separate the pathsum into contributions from the bulk, intermediate contributions that "communicate from bulk to boundary" and purely boundary terms. We then take the semi-classical limit of the bulk part of the path-sum and discuss the resulting action. In Section 4 we make some comments on the resulting split path-sum and the significance of the various terms.

\section{Ponzano-Regge gravity in the presence of boundaries}

The original Ponzano-Regge model [11], associated with the partition function of 3dimensional Euclidean gravity for a closed simplicial manifold $M^{3}$, can be extended to deal with manifolds with a non-empty boundary $\left(M^{3}, \partial M^{3}\right.$ ) (where $\partial M^{3}$ could be the disjoint union of a finite number of components). The basic idea is to consider the triangulation induced on the boundary by the bulk simplicial decomposition of the three-manifold since the tetrahedra that have one or more of their triangular faces in common with the boundary induce a boundary triangulation. The weight of the path sum associated with this boundary triangulation can be determined in a variety of ways. Via the canonical formalism whereby one considers a two-manifold that is evolving in Euclidean time [12]. Otherwise we can use a general topological construction [13, 14], in which one enforces invariance of the path sum including boundary under the discretized version of diffeomorphisms [15]. Finally, via the discretization of the corresponding BF (first-order formalism for three-dimensional gravity) theory, imposing suitable boundary conditions on the path integral [16]. In each of these cases, the path sum includes sum over the boundary spins, and thus the boundary geometry has identical weights. In the case of [12] and [16], this boundary sum remains also a function of the connection since boundary conditions were chosen such that the connection was fixed on the boundary.

We give in the rest of this section a brief review of these results for the path sum with fluctuating boundary. For simplicity we will take the connection to be trivial, although in general a global gauge choice of this type cannot be made due to possible non-trivial holonomy along one-cycles in the boundary two-fold.

Let $\left(M^{3}, \partial M^{3}\right)$ be a Piecewise Linear $(P L)$ pair of a fixed topological type, and consider a particular triangulation $\left(T^{3}(J), \partial T^{3}(j ; m)\right)$ with edges, faces and tetrahedra labelled by suitable elements of the Racah-Wigner algebra of $S U(2)$ (or of its $q$-deformed counterpart, $q$ a root of unity [17]). More precisely, we weight the contribution of any such triangulation to the partition function (or state sum) $Z\left[\left(M^{3}, \partial M^{3}\right)\right]$ by introducing the following functional [13]:

$$
\begin{aligned}
& Z\left[\left(T^{3}(J), \partial T^{3}(j ; m)\right) \rightarrow\left(M^{3}, \partial M^{3}\right) ; L\right] \equiv Z\left[\left(T^{3}, \partial T^{3}\right) ; L\right]= \\
& \Lambda(L)^{-N_{0}} \prod_{1}^{N_{1}}(-1)^{2 J}(2 J+1) \prod_{1}^{N_{3}}(-1)^{\sum J} \quad\{6 j\}(J) \\
& \Lambda(L)^{-n_{0}} \prod_{1}^{n_{1}}(-1)^{2 j}(2 j+1) \prod_{1}^{N_{3}^{F}}(-1)^{\sum(J+j)}\{6 j\}(J, j) \\
& \prod_{1}^{n_{2}}(-1)^{\left(\sum m\right) / 2}[3 j m] .
\end{aligned}
$$


Here $N_{0}, N_{1}, N_{3}$ denote the number of vertices, edges and tetrahedra which lie entirely in the interior of $T^{3}\left(\operatorname{Int} T^{3}\right)$, while $n_{0}, n_{1}, n_{2}$ are the number of vertices, edges and triangular faces in $\partial T^{3} ; N_{3}^{F}$ is the number of tetrahedra which have faces in $\partial T^{3}$.

The set of spin variables $\{J\}$ labels edges in $\operatorname{Int} T^{3}$, while the set $\{j\}$ is associated with edges in $\partial T^{3}$; each spin variable runs over $\{0,1 / 2,1, \ldots\}$. The cut-off $L$ plays here the same role as in Ponzano-Regge asymptotic formula [11], i.e. $\Lambda(L)=4 L^{3} / 3 c$ (c an arbitrary constant), and all spin variables in (1) are bounded above by $L$.

Each of the symbol in (11) has a precise group theoretical and geometrical meaning, namely:

- $(2 J+1)$ [respectively $(2 j+1)]$, the dimension of the $J$-th [respectively $j-$ th] irreducible representation of $S U(2)$, gives the contribution to the measure of each edge belonging to $\operatorname{IntT}^{3}$ [respectively $\left.\partial T^{3}\right]$.

- The Racah-Wigner $6 j$ symbol

$$
\{6 j\}(J) \equiv\left\{\begin{array}{ccc}
J_{1} & J_{2} & J_{3} \\
J_{4} & J_{5} & J_{6}
\end{array}\right\}
$$

represents, apart from the phase factor $(-1)^{\sum J}, \sum J=J_{1}+J_{2}+\ldots+J_{6}$, a tetrahedron in $\operatorname{IntT}^{3}$.

The notation $\{6 j\}(J, j)$ stands for a tetrahedron which has some of its faces in $\partial T^{3}$, and thus the entries $\{J, j\}$ of such symbol depend on its place in the triangulation.

- The Wigner $3 j m$ symbol

$$
[3 j m] \equiv\left[\begin{array}{ccc}
j_{1} & j_{2} & j_{3} \\
m_{1} & m_{2} & -m_{3}
\end{array}\right],
$$

apart from the phase factor $(-1)^{\left(\sum m\right) / 2}, \sum m=m_{1}+m_{2}+m_{3}$, is associated with a triangular face in $\partial T^{3}$, and each $m$-variable (representing the projection of the corresponding $j$ with respect to a fixed quantization axis) satisfies the usual requirement $-j \leq m \leq j$ in integer steps.

The partition function to be associated with the manifold $\left(M^{3}, \partial M^{3}\right)$ is found by summing the contributions of the type (1) over all admissible angular momentum configurations for the simplicial decomposition $\left(T^{3}(J), \partial T^{3}(j ; m)\right) \rightarrow\left(M^{3}, \partial M^{3}\right)$, under the condition that all spin variables $\{J, j\}$ - together with the $m$ 's - are bounded above by a fixed $L$. In the spirit of the Ponzano-Regge approach, in order to remove the cut-off we formally take the limit $L \rightarrow \infty$ of the state sum, and thus the expression of the "regularized" partition function can be formally written as:

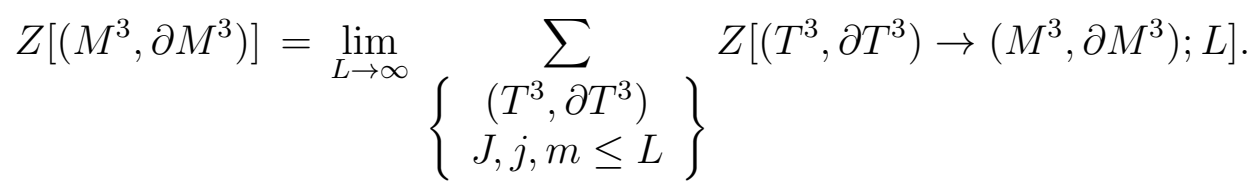

This state sum reduces to the Ponzano-Regge functional in the case $\partial M^{3}=\emptyset$, while we would recover the expression found in [12] if we keep fixed the triangulation $\overline{\partial T^{3}}$ on the 
boundary manifold, i.e. for $\left(M^{3}, \partial M^{3}=\overline{\partial T^{3}}\right)$.

In the asymptotical limit (all spin variables large) (4) represents the semiclassical partition function of Euclidean gravity in the presence of a boundary with the action discretized according to the Regge prescription [18], ( $c f r$. [19] for the extension of Regge Calculus to manifolds with boundary). More precisely, for a triangulation $\left(T^{3}, \partial T^{3}\right)$ the action contains the Einstein-Regge action of the bulk, namely $\mathcal{I}\left(\operatorname{Int} T^{3}\right) \propto \sum_{J}(J+1 / 2) \theta$ (where $\theta$ 's are the deficit angles), and a suitable contribution due to the boundary $\partial T^{3}$.

To discuss the role of the boundary terms in the partition function (बi) we refer now to [20] (see also [16, 14, 12]), where the state sum model induced on the closed boundary manifold $\partial M^{3} \equiv M^{2}$ was derived. It turns out that each triangular face in a triangulation $T^{2}\left(j ; m, m^{\prime}\right) \rightarrow M^{2}$ has to be associated with the following product of two $3 j m$ symbols

$$
(-1)^{\sum_{s=1}^{3}\left(m_{s}+m_{s}^{\prime}\right) / 2}\left[\begin{array}{ccc}
j_{1} & j_{2} & j_{3} \\
m_{1} & m_{2} & -m_{3}
\end{array}\right]\left[\begin{array}{ccc}
j_{1} & j_{2} & j_{3} \\
m_{1}^{\prime} & m_{2}^{\prime} & -m_{3}^{\prime}
\end{array}\right] .
$$

Here $\left\{m_{s}\right\}$ and $\left\{m_{s}^{\prime}\right\}$ are two different sets of momentum projections associated with the same angular momentum variables $\left\{j_{s}\right\}$, and $-j_{s} \leq m_{s}, m_{s}^{\prime} \leq j_{s} \forall s=1,2,3$.

The expression of the functional associated with a particular triangulation $T^{2}\left(j ; m, m^{\prime}\right)$ is

$$
\begin{aligned}
Z\left[T^{2}\left(j ; m, m^{\prime}\right) \rightarrow\right. & \left.M^{2} ; L\right] \equiv Z\left[T^{2} ; L\right]= \\
& \Lambda(L)^{-n_{0}} \prod_{A=1}^{n_{1}}\left(2 j_{A}+1\right)(-1)^{2 j_{A}}(-1)^{-m_{A}-m_{A}^{\prime}} \\
& \prod_{B=1}^{n_{2}}\left[\begin{array}{ccc}
j_{1} & j_{2} & j_{3} \\
m_{1} & m_{2} & -m_{3}
\end{array}\right]_{B}\left[\begin{array}{ccc}
j_{1} & j_{2} & j_{3} \\
m_{1}^{\prime} & m_{2}^{\prime} & -m_{3}^{\prime}
\end{array}\right]_{B},
\end{aligned}
$$

where $n_{0}, n_{1}, n_{2}$ are the numbers of vertices, edges and triangles in $T^{2}\left(j ; m, m^{\prime}\right)$, respectively. Summing over all admissible triangulations we get the partition function of the closed 2-dimensional theory which reads

$$
Z\left[M^{2}\right]=\lim _{L \rightarrow \infty} \sum_{\left\{T^{2} ; j, m, m^{\prime} \leq L\right\}} Z\left[T^{2} ; L\right],
$$

where the regularization is carried out according to the previous prescription. This expression can be evaluated in a staightforward way, providing the expression

$$
Z\left[M^{2}\right]=\Lambda(L) \Lambda(L)^{-\chi\left(M^{2}\right)},
$$

where $\chi\left(M^{2}\right)$ is the Euler character of the manifold $M^{2}$.

Thus the partition function of the 2-dimensional closed model induced on the boundary by (4) contains the only topological invariant which is significant in the present context (recall also that the Regge action in dimension 2 is just $\chi\left(M^{2}\right)$ ).

Note that the regularization prescription used above is ill defined in general as there is no natural way to remove the cut-off. The naturally regularized counterpart of the closed Ponzano-Regge model is the quantum invariant introduced in [17 which turns out to be related to a double Chern-Simons-Witten theory at level $k$ where $\exp (\pi i / k)=q$ is the deformation parameter. Moreover, the limit for $q \rightarrow 1$ of the Turaev-Viro functional corresponds to a semiclassical partition function containing an Einstein-Regge term plus 
a volume term with a positive cosmological constant related to $k$ [28] (cfr. also [21, 22] on these issues). In [13, [14 the $q$-deformed counterparts of the state sums (4) and (8) have been defined, and in particular (8) corresponds to the quantum invariant

$$
Z\left[M^{2}\right]_{q}=w_{q}^{2} w_{q}^{-2 \chi\left(M^{2}\right)}
$$

where $w_{q}^{2}=(-2 k) /\left(q-q^{-1}\right)^{2}$.

The state sums associated with simplicial dissections of 3-manifolds based on the recoupling theory of quantum angular momenta have a rich topological structure which is non trivially implemented by exploiting a suitable set of operations on simplices (topological moves). In general, given two compact $d$-dimensional simplicial $P L$-manifolds (with or without boundaries) Pachner proved that they are $P L$-homeomorphic if and only if their underlying triangulations are related to each other either by a finite set of bistellar moves (in the closed case, 23]) or by a finite set of elementary shellings (in the case with boundary, [15]). Since in $d \leq 3$ the $P L$-type and the topological equivalence class of any compact manifold are in $1-1$ correspondence, in the present context we can simply speak of "topological equivalent" or "homeomorphic" manifolds.

The next step consists in recognizing that all the moves we are referring to can be translated into algebraic identities which are encoded in the structure of the partition functions written above.

Recall that in the closed 3-dimensional case (see [11], [17]) the bistellar moves can be expressed algebraically in terms of the Biedenharn-Elliott identity (representing the moves [2 tetrahedra joined along a common face $] \leftrightarrow$ [3 tetrahedra joined along a common edge]) and of both the B-E identity and the orthogonality conditions for $6 j$ symbols, which represent the barycentic move together with its inverse, namely $[1$ tetrahedron $] \leftrightarrow[4$ tetrahedra]. Thus the Ponzano-Regge functional, namely (4) for $\partial M^{3}=\emptyset$, is formally a topological invariant (and the Turaev-Viro state sum is in fact a well-defined topological invariant).

In [20] the identities representing the bistellar moves in $d=2$ have been established (and they will be given explicitly in the next section). They are associated with the flip move [2 triangles] $\rightarrow$ [2 triangles] (a pair of triangles joined to form a quadrilateral are transformed into two triangles joined along the other diagonal) and with the barycentric move and its inverse, namely [ 1 triangle $] \leftrightarrow$ [3 triangles]. Then the fact that (7) is actually a topological invariant can be recognized on the basis of its manifest invariance under any finite set of such moves.

Turning now to the case of $\left(M^{3}, \partial M^{3}\right)$, the topological transformations that have to be taken into account are the elementary shellings and their inverse moves introduced in [15. These operations involve the cancellation of one tetrahedron at a time from a given triangulation $\left(T^{3}(J), \partial T^{3}(j ; m)\right) \rightarrow\left(M^{3}, \partial M^{3}\right)$. In order to be deleted, the tetrahedron must have some of its 2-dimensional faces lying on the boundary $\partial T^{3}$. Moreover, for each elementary shelling there exists an inverse move which corresponds to the attachment of a new tetrahedron to a suitable component in $\partial T^{3}$. In [13 the identities corresponding to such moves have been found, and the expression of the state sum given in (4) and (11) is actually manifestly invariant under any finite set of such moves. The conclusion is that, owing to Pachner's results, (4) is a topological $(P L)$ invariant of the pair $\left(M^{3}, \partial M^{3}\right)$. Notice also that, since $Z\left[\left(M^{3}, \partial M^{3}\right)\right]$ reduces to the Ponzano-Regge partition function 
in the case $\partial M^{3}=\emptyset$, it is automatically invariant also under bistellar moves in the bulk Int $M^{3}$.

\section{Projecting on the boundary and the decoupling limit}

In the spirit of the holographic principle we would like to decouple - or more properly disentangle - the theory living on the boundary from the bulk gravity theory. We therefore look for some way of projecting the theory described in the previous section onto the boundary. Notice that the 2-dimensional state sum given in (7) is not generated in this way since it simply represents a theory obtained from the 3-dimensional functional (1) by restricting it to the boundary and by summing freely over all triangulations of the surface $\partial M^{3}=M^{2}$, up to regularization.

To set up a true decoupling procedure one has to examine more carefully the structure of the functional (国) describing the state of a single triangulation $\left(T^{3}(J), \partial T^{3}(j ; m)\right)$. In particular, one expects to recognize bulk pieces, boundary pieces and interaction terms bulk-boundary. Bulk pieces are going to describe the fluctuations of the spins $J$ 's in the interior of the manifold, boundary pieces the fluctuations of the spins $j$ 's (and of their corresponding $m$ 's) on the boundary, and the interaction terms the "coupling" between such bulk-boundary fluctuations.

The interaction bulk-boundary is clearly given by those tetrahedra which have some of their components on the boundary triangulation, and in particular by terms of the type $\{6 j\}(J, j)$ in (11). Such symbols may have in principle a varying number of spins of type $J$ or $j$. But, as discussed at the end of the previous section, we can take advantage of the invariance of $Z\left[\left(M^{3}, \partial M^{3}\right)\right]$ in (4) under elementary shellings. In particular we can always transform a given triangulation $\left(T^{3}(J), \partial T^{3}(j ; m)\right) \rightarrow\left(M^{3}, \partial M^{3}\right)$ in such a way that each tetrahedron which shares with the boundary a number of faces has actually only one face in $\partial T^{3}$. Call the resulting dissection standard triangulation to be denoted from now on by $\left(T^{3}, \partial T^{3}\right)_{s t}$.

Note however that in a standard triangulation there are two distinct types of tetrahedra having edges in $\left(\partial T^{3}\right)_{s t}$ :

- The tetrahedra $\left\{\sigma_{F}\right\}$ which are involved in the definition of the standard triangulation itself, namely the ones which share with the boundary one of their faces and the corresponding three edges. Their number $N_{3}^{F}$ is equal to the number of triangular faces in $\left(\partial T^{3}\right)_{s t}$, which have been denoted by $n_{2}$ in (四), namely we have

$$
N_{3}^{F}=n_{2} \text {. }
$$

- The tetrahedra $\left\{\sigma_{E}\right\}$ which have exactly one edge in $\left(\partial T^{3}\right)_{s t}$ and no corresponding face (there could be a varying number of such tetrahedra for any edge in $\left(\partial T^{3}\right)_{s t}$ ). Denote their total number by $N_{3}^{E}$.

We shall call the $\left\{\sigma_{F}, \sigma_{E}\right\}$ coupling tetrahedra.

The $\{6 j\}(J, j)$ symbols to be associated with these types of coupling tetrahedra in the state functional of a standard triangulation can be always cast - by making use of the symmetry properties of the $6 j$ symbols - in two particular forms 


$$
\sigma_{F} \longleftrightarrow(-1)^{\sum J+\sum j}\left\{\begin{array}{lll}
j_{1} & j_{2} & j_{3} \\
J_{1} & J_{2} & J_{3}
\end{array}\right\} \doteq(-1)^{\sum J+\sum j}\{6 j\}\left(\sigma_{F}\right),
$$

where the phase factor is explicitly given by $\sum_{i=1}^{3} J_{i}+\sum_{i=1}^{3} j_{i}$, and

$$
\sigma_{E} \longleftrightarrow(-1)^{\sum J+j}\left\{\begin{array}{lll}
J_{1} & j & J_{2} \\
J_{3} & J_{4} & J_{5}
\end{array}\right\} \doteq(-1)^{\sum J+j}\{6 j\}\left(\sigma_{E}\right)
$$

with the phase factor given by $\sum_{i=1}^{5} J_{i}$.

Thus, if we denote by

$$
\begin{aligned}
& N_{1}^{F}=\text { number of edges of type } J \text { in }\left\{\sigma_{F}\right\} \\
& N_{1}^{E}=\text { number of edges of type } J \text { in }\left\{\sigma_{E}\right\}
\end{aligned}
$$

we can rewrite the state functional (1) for a standard triangulation $\left(T^{3}, \partial T^{3}\right)_{s t}$ by setting

$$
\begin{aligned}
& Z\left[\left(T^{3}(J), \partial T^{3}(j ; m)\right)_{s t} ; L\right] \equiv Z\left[\left(T^{3}, \partial T^{3}\right)_{s t} ; L\right]= \\
& \Lambda(L)^{-N_{0}} \prod_{1}^{N_{1}-N_{1}^{F *}-N_{1}^{E *}}(-1)^{2 J}(2 J+1) \prod_{1}^{N_{3}-N_{3}^{E}}(-1)^{\sum J}\{6 j\}(J) \\
& \prod_{1}^{N_{1}^{F *}}(-1)^{2 J^{F}}\left(2 J^{F}+1\right) \prod_{1}^{N_{1}^{E *}}(-1)^{2 J^{E}}\left(2 J^{E}+1\right) \\
& \Lambda(L)^{-n_{0}} \prod_{1}^{n_{1}}(-1)^{2 j}(2 j+1) \\
& \prod_{1}^{n_{2}}(-1)^{\sum J+\sum j}\{6 j\}\left(\sigma_{F}\right)(-1)^{\left(\sum m\right) / 2}[3 j m] \\
& \prod_{1}^{N_{3}^{E}}(-1)^{\sum J+j}\{6 j\}\left(\sigma_{E}\right) .
\end{aligned}
$$

where the edges of the coupling tetrahedra $\left\{\sigma_{F}, \sigma_{E}\right\}$ have been labelled by $F$ and $E$, respectively. We have introduced $N_{1}^{F *}<N_{1}^{F}$ and $N_{1}^{E *}<N_{1}^{E}$ as there are only $N_{1}^{F *}+N_{1}^{E *}$ independent edges of type $\mathrm{J}$ in $\left\{\sigma_{F}, \sigma_{E}\right\}$ due to the obvious identification of edges in common to more than one of these coupling tetrahedra.

Ponzano-Regge gravity is a discretized counterpart of a second order theory in which the variables associated with the edges play the role of the metric tensor [18]. Thus, in the spirit of an holographic scenario, one has to take a suitable limit on some "metric variables" living close to the boundary in order to be able to decouple the boundary theory from the bulk. The collection of the coupling tetrahedra $\left\{\sigma_{F}, \sigma_{E}\right\}$ is obviously a natural candidate to play this role. However, we easily recognize that there is a further class of tetrahedra living close to the boundary, namely those tetrahedra with edges in IntT $^{3}$, but which have one of their vertices in $\left(\partial T^{3}\right)_{s t}$ (the weights of these vertices are included in the factor $\Lambda(L)^{-n_{0}}$ of (15)). Denote this new collection by $\left\{\sigma_{V}\right\}$ and call them coupling tetrahedra too. Their number runs, say, from 1 to $N_{3}^{V}$, and each of them corresponds to a $6 j$ symbol of the form 


$$
\sigma_{V} \longleftrightarrow(-1)^{\sum J}\left\{\begin{array}{lll}
J_{1} & J_{2} & J_{3} \\
J_{4} & J_{5} & J_{6}
\end{array}\right\} \doteq(-1)^{\sum J}\{6 j\}\left(\sigma_{V}\right)
$$

where no confusion should arise with the generic symbol introduced in (2). Then, if we denote by

$$
N_{1}^{V}=\text { number of edges in }\left\{\sigma_{V}\right\}
$$

the first two groups of terms in the right-hand side of (15) can be rewritten as

$$
\begin{aligned}
\Lambda(L)^{-N_{0}} & \prod_{1}^{N_{1}-N_{1}^{F *}-N_{1}^{E *}-N_{1}^{V *}}(-1)^{2 J}(2 J+1) \prod_{1}^{N_{3}-N_{3}^{E}-N_{3}^{V}}(-1)^{\sum J}\{6 j\}(J) \\
& \prod_{1}^{N_{1}^{F *}}(-1)^{2 J^{F}}\left(2 J^{F}+1\right) \prod_{1}^{N_{1}^{E *}(-1)^{2 J^{E}}\left(2 J^{E}+1\right)} \prod_{1}^{N_{1}^{V *}(-1)^{2 J^{V}}}\left(2 J^{V}+1\right) \doteq \\
& \Lambda(L)^{-N_{0}} \prod_{1}^{N_{1}-N_{1}^{*}}(-1)^{2 J}(2 J+1) \prod_{1}^{N_{3}-N_{3}^{E}-N_{3}^{V}}(-1)^{\sum J}\{6 j\}(J) \\
& \prod_{1}^{N_{1}^{*}}(-1)^{2 J}(2 J+1),
\end{aligned}
$$

where $N_{1}^{V *}$ again takes into account the additional necessary identifications of edges between the tetrahedra $\sigma_{V}$ and the tetrahedrae $\left\{\sigma_{F}, \sigma_{E}\right\}$. On the right-hand side of (18) we have then defined

$$
N_{1}^{*} \doteq \text { number of edges in }\left(\operatorname{Int} T^{3}\right)_{s t} \backslash\left(\left\{\sigma_{F}\right\} \cup\left\{\sigma_{E}\right\} \cup\left\{\sigma_{V}\right\}\right) .
$$

The role of the weights of such edges, labelled again by $J$ to simplify the notation, will be discussed below.

In order to set up a decoupling procedure in (15) (taking into account (18)), we have to keep fixed all labels $\{j, m\}$ together with the $N_{1}-N_{1}^{*}$ variables of type $J$, while rescaling the $N_{1}^{*} J$-variables belonging to the coupling tetrahedra by the same factor $R$. More precisely, we have different asymptotic expressions for each type of coupling tetrahedra (see [11], 24] and the original references therein), namely

- For a tetrahedron $\sigma_{F}$ we get

$$
\{6 j\}\left(\sigma_{F}, R\right) \equiv\left\{\begin{array}{ccc}
j_{1} & j_{2} & j_{3} \\
J_{1}+R & J_{2}+R & J_{3}+R
\end{array}\right\} \underset{R \gg 1}{\longrightarrow} \frac{(-1)^{\Phi}}{\sqrt{2 R}}\left[\begin{array}{ccc}
j_{1} & j_{2} & j_{3} \\
\mu_{1} & \mu_{2} & \mu_{3}
\end{array}\right]
$$

where $\Phi=j_{1}+j_{2}+j_{3}+2\left(J_{1}+J_{2}+J_{3}\right)$. Such an expression tells us that by rescaling the three $J$-variables by a large $R$, this $6 j$ symbol goes into a weighted Wigner $3 j m$ symbol with the same $j$ 's, and with momentum projections depending on differences of $J$ 's according to

$$
\left\{\begin{array}{l}
\mu_{1}=J_{2}-J_{3} \\
\mu_{2}=J_{3}-J_{1} \\
\mu_{3}=J_{1}-J_{2}
\end{array}\right.
$$


- For a tetrahedron $\sigma_{E}$ we get

$$
\{6 j\}\left(\sigma_{E}, R\right) \equiv\left\{\begin{array}{ccc}
J_{1}+R & j & J_{2}+R \\
J_{3}+R & J_{4}+R & J_{5}+R
\end{array}\right\} \quad \longrightarrow \gg 1 \frac{(-1)^{\Psi}}{2 R} d_{\nu_{2} \nu_{3}}^{j}(\theta),
$$

where $\Psi=3 J_{1}+j+2\left(J_{2}+J_{3}+J_{4}+J_{5}\right)+\nu_{1}$ and $\theta$ is the angle between the the edge labelled by $j$ and the quantization axis. Thus we get that the asymptotic behavior of such a symbol goes like a product of a Kronecker $\delta$ and a Wigner $d$-function with entries given by

$$
\left\{\begin{array}{l}
\nu_{1}=J_{3}-J_{4}, \\
\nu_{2}=J_{5}-J_{3}, \\
\nu_{3}=J_{2}-J_{1} .
\end{array}\right.
$$

- Finally, the asymptotic expression for a tetrahedron $\sigma_{V}$ is nothing but the original Ponzano-Regge formula [11], namely

$$
\begin{array}{r}
\{6 j\}\left(\sigma_{V}, R\right) \equiv\left\{\begin{array}{lll}
J_{1}+R & J_{2}+R & J_{2}+R \\
J_{4}+R & J_{5}+R & J_{6}+R
\end{array}\right\} \quad \overrightarrow{R \gg 1} \\
\left(12 \pi \mathcal{V}\left(\sigma_{V}\right)\right)^{-1 / 2} \exp \left\{i\left(\sum_{\alpha=1}^{6} l_{\alpha} \theta_{\alpha}+\pi / 4\right)\right\} .
\end{array}
$$

$\mathcal{V}\left(\sigma_{V}\right)$ is the Euclidean volume of the tetrahedron spanned by the six edges $\left\{l_{\alpha}\right\}$, $l_{\alpha}=J_{\alpha}+1 / 2$ and $\theta_{\alpha}$ is the angle between the outward normals to the faces which share $l_{\alpha}$ (these angles can be obviously expressed in terms of the $J$ 's). Note that the sum under the exponential is the Regge action for the tetrahedron $\sigma_{V}$.

Since we can freely choose the decoupling parameter $R \gg J(\leq L)$ at this level, we carry out the rescaling both on the three types of $6 j$ symbols according to (20), (22), (24) and on the $N_{1}^{*}$ phase factors and weights $(2 J+1) \sim(2 R)$ in (15) and (18). Then the functional which turns out to be associated with the resulting configuration is

$$
\begin{aligned}
& Z\left[\left(T^{3}, \partial T^{3}\right)_{s t} ; R \gg 1 ; L\right]= \\
& \Lambda(L)^{-N_{0}} \prod_{1}^{N_{1}-N_{1}^{*}}(-1)^{2 J}(2 J+1) \prod_{1}^{N_{3}-N_{3}^{E}-N_{3}^{V}}(-1)^{\sum J}\{6 j\}(J) \\
& \prod_{1}^{N_{1}^{*}}(-1)^{2 R}(2 R) \Lambda(L)^{-n_{0}} \prod_{1}^{n_{1}}(-1)^{2 j}(2 j+1) \\
& \prod_{1}^{n_{2}}(2 R)^{-1 / 2}(-1)^{\sum(J+R)+\sum j+\Phi}(-1)^{\left(\sum m\right) / 2}[3 j \mu][3 j m] \\
& \prod_{1}^{N_{3}^{E}}(2 R)^{-1}(-1)^{\sum(J+R)+j+\Psi} d_{\nu_{2} \nu_{3}}^{j}(\theta)
\end{aligned}
$$




$$
\prod_{1}^{N_{3}^{V}}(2 R)^{-3 / 2}(-1)^{\sum(J+R)} \exp \left\{i\left(\sum_{\alpha=1}^{6} l_{\alpha} \theta_{\alpha}+\pi / 4\right)\right\},
$$

where each term containing $(2 R)^{-3 / 2}$ is the approximation for the corresponding volume factor in (24).

This expression is an almost factorized product of two groups of terms

i) A Ponzano-Regge-like state functional for a 3-dimensional bulk triangulation to be closed with boundary terms depending on some of the $N_{1}^{*}$ fixed edges $J+R$, (see below for more details). The sum of this state functional over all possible triangulations with fixed labels on the boundary (up to regularization) will provide in the bulk a family of 3-gravity partition functions depending on the decoupling parameter $R$.

ii) A second functional, represented by the remaining terms in (25), containing contributions resembling the state functional for a 2-dimensional closed triangulation given in (7) (i.e. a pair of Wigner $3 \mathrm{jm}$ symbols for each triangular face).

Referring to $i)$, we recognize that the topological union of the coupling tetrahedra, $\left(\left\{\sigma_{F}\right\} \cup\left\{\sigma_{E}\right\} \cup\left\{\sigma_{V}\right\}\right)$, fills in a thick shell close to the boundary $\left(\partial T^{3}\right)_{s t}$. The linear extension of such a shell is of the order of the decoupling parameter $R$, and some of the $N_{1}^{*}$ edges considered in (18) happen to lie on the boundary of the 3-dimensional triangulation

$$
\tilde{T}^{3} \doteq\left(\operatorname{Int} T^{3}\right)_{s t} \backslash\left(\left\{\sigma_{F}\right\} \cup\left\{\sigma_{E}\right\} \cup\left\{\sigma_{V}\right\}\right) .
$$

For each value $R \gg 1$ of the decoupling parameter, denote by $\Sigma^{\text {in }}(R)$ the fixed 2dimensional triangulation which closes up (26) giving rise to the pair

$$
\left(\tilde{T}^{3}, \Sigma^{i n}(R)\right) \text {. }
$$

Then any such a pair is topologically equivalent to the original pair $\left(T^{3}, \partial T^{3}\right)_{s t}$ as can be easily seen by exploiting the invariance under elementary shellings discussed at the end of Section 2. Moreover, with respect to $\left(T^{3}, \partial T^{3}\right)_{s t}, \Sigma^{i n}(R)$ represents an inner boundary with

$$
\Sigma^{i n}(R) \cap\left(\partial T^{3}\right)_{s t}=\emptyset .
$$

For what concerns the state functional to be associated with (27), note that only contributions from the edges in $\Sigma^{i n}(R)$ - and not from its faces - have to be taken into account. This is due to the fact that the contribution to the state functional of an edge on a fixed boundary amounts simply to $(-1)^{J}(2 J+1)^{1 / 2}$ [12], which further reduces to $(-1)^{R}(2 R)^{1 / 2}$ in our case.

If we now select

$$
N_{1}\left(\Sigma^{i n}(R)\right) \doteq \text { number of edges in } \Sigma^{i n}(R)
$$

out of the $N_{1}^{*}$ edges in (18), by collecting from (25) the terms from the bulk, we get

$$
\begin{aligned}
& Z\left[\left(\tilde{T}^{3}, \Sigma^{i n}(R)\right) ; L\right]= \\
& \Lambda(L)^{-\left(N_{0}-N_{0}\left(\Sigma^{i n}\right)\right)} \prod_{1}^{N_{1}-N_{1}^{*}}(-1)^{2 J}(2 J+1) \prod_{1}^{N_{3}}(-1)^{\sum J}\{6 j\}(J)
\end{aligned}
$$




$$
\prod_{1}^{N_{1}\left(\Sigma^{i n}\right)}(-1)^{R}(2 R)^{1 / 2}
$$

Notice that here we are forced to include only $\left(N_{0}-N_{0}\left(\sum^{i n}\right)\right)$ vertex weights, $N_{0}\left(\Sigma^{i n}\right)=$ (number of vertices of the inner boundary), namely those vertices which are actually in the new $\operatorname{Int} \tilde{T}^{3}$.

As remarked before, we have now the possibility of getting at once the partition function of the bulk on applying the standard formal procedure in the case of a fixed boundary, i.e.

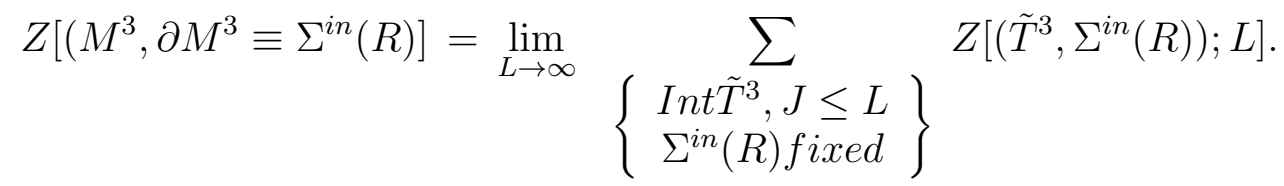

This family of Euclidean 3-gravity partition functions encodes information about the decoupling parameter $R$ in its fixed boundary, while free fluctuations of all the other spin variables in the bulk are obviously allowed. In the decoupling limit to be discussed below, the state sum (31) should be sharply peaked on (semi)classical configurations, namely on states $(30)$ in which all spin variables $J$ in $\operatorname{IntT}^{3}$ have been rescaled by choosing some finite $L \gg 1$, with $L<R$. But such conditions do not affect the "topological character" of (31), since Ponzano and Regge showed that also the asymptotic $6 j$ symbols satisfy all the algebraic identities [11], and thus the bistellar moves in $\operatorname{IntT}^{3}$ can work as well as before.

Coming back now to the factorization of the state functional (25), and taking into account (30), we can formally write

$$
\begin{aligned}
& Z\left[\left(T^{3}, \partial T^{3}\right)_{s t} ; R \gg 1 ; L\right]= \\
& Z\left[\left(\tilde{T}^{3}, \Sigma^{\text {in }}(R)\right) ; L\right] \mathcal{P}\left(\Sigma^{\text {in }}, \Sigma^{\text {out }} ; R\right) Z^{\text {hol }}\left[\Sigma^{\text {out }} ; L\right],
\end{aligned}
$$

where we have set

$$
\left(\partial T^{3}\right)_{s t} \equiv \Sigma^{o u t}
$$

to denote the (fluctuating) outer boundary, in agreement with the notation $\Sigma^{\text {in }}$ for the (frozen) inner boundary.

The expressions of the last two terms on the right-hand side of (32) can be recognized from (25), taking into account (29), (33) and (30). Thus

- In the projection map

$$
\begin{gathered}
\mathcal{P}\left(\Sigma^{\text {in }}, \Sigma^{\text {out }} ; R\right) \doteq \\
\prod_{1}^{N_{1}^{*}-N_{1}\left(\Sigma^{i n}\right)}(2 R) \prod_{1}^{N_{1}\left(\Sigma^{i n}\right)}(2 R)^{1 / 2} \prod_{1}^{n_{2}}(2 R)^{-1 / 2} \prod_{1}^{N_{3}^{E}}(2 R)^{-1} \prod_{1}^{N_{3}^{V}}(2 R)^{-3 / 2}= \\
(2 R)^{N_{1}^{*}-\frac{1}{2} N_{1}\left(\Sigma^{i n}\right)}\left(\frac{1}{2 R}\right)^{\frac{1}{2} n_{2}}\left(\frac{1}{2 R}\right)^{N_{3}^{E}}\left(\frac{1}{2 R}\right)^{\frac{3}{2} N_{3}^{V}}
\end{gathered}
$$


we collect all the terms of (25) which depend explicitly from $R$ (the phase factors containing $R$ can be dropped e.g. by choosing an even $R$ ).

- The remaining terms of (25), not yet considered, are

$$
\begin{aligned}
Z^{\text {hol }\left[\Sigma^{\text {out }} ; L\right]} & = \\
& \Lambda(L)^{-n_{0}-N_{0}\left(\Sigma^{i n}\right)} \prod_{1}^{n_{1}}(-1)^{2 j}(2 j+1) \\
& \prod_{1}^{n_{2}}(-1)^{\sum J+\sum j+\Phi}(-1)^{\left(\sum m\right) / 2}[3 j \mu] \quad[3 j m] \\
& \prod_{1}^{N_{3}^{E}}(-1)^{\sum J+j+\Psi} d_{\nu_{2} \nu_{3}}^{j}(\theta) \\
& \prod_{1}^{N_{3}^{V}}(-1)^{\sum J} \exp \left\{i\left(\sum_{\alpha=1}^{6} l_{\alpha} \theta_{\alpha}+\pi / 4\right)\right\}
\end{aligned}
$$

where the extra-factor due to the vertices of $\Sigma^{i n}$ - which compensates the modification of the weights in (30) - has been included. This expression represents the functional to be associated with a holographic state generated by disentangling the external boundary from the original 3-dimensional theory.

As a first remark, note that the projection map includes combinatorial quantities related not only to the topology of the manifold $\left(T^{3}, \partial T^{3}\right)_{s t}$, but also to the triangulations of $\Sigma^{\text {in }}$, $\Sigma^{\text {out }}$ and of the shell between them. Thus, in particular, the numbers $N_{1}^{*}, N_{1}\left(\Sigma^{i n}\right)$, $n_{2} \equiv n_{2}\left(\sum^{\text {out }}\right), N_{3}^{E}, N_{3}^{V}$ in (34) cannot be related to each other in a unique way unless we choose a priori the topological type and the triangulations.

Anyway, we may say that the onset of a decoupling regime is achieved when the value of the limit

$$
\lim _{R \rightarrow \infty} \mathcal{P}\left(\Sigma^{\text {in }}, \Sigma^{\text {out }} ; R\right)
$$

is small as compared with the other functionals appearing in the factorization (32). In other words, we require that the gravitational contribution of the shell made up by the coupling tetrahedra is negligible when approaching the large $R$-limit both with respect to the bulk contribution (30) and also with respect to the residual functional (35) surviving on the external boundary. Such a kind of behavior can be translated into suitable sets of selection rules (or more properly, in holographic language, a sort of "screen map" [2]) on the triangulations involved in (34). Indeed, given a topological type $\left(M^{3}, \partial M^{3}\right)$, we can in principle select - by making use of both bistellar moves in the interior of $M^{3}$ and shellings and/or inverse shellings in the boundary - exactly those standard triangulations which satisfy the constraints, and in the decoupling limit the bulk-boundary transfer process is efficiently performed only by such classes of triangulations. We shall return to the physical interpretation of this point in the next section.

An inspection of the holographic state functional (35) shows that it is not independent of the triangulation $\Sigma^{\text {out }}$. This feature is an obvious consequence of the factorizazion 
prescription (32), which breaks the topological invariance of the original $Z\left[\left(T^{3}, \partial T^{3}\right)_{s t}\right.$ $\left.\rightarrow\left(M^{3}, \partial M^{3}\right) ; L\right]$. Even if such an invariance can be restored in the bulk according to the procedure given in (31), by comparing (35) with the bidimensional functional (6) we see that in the present case a number of new significant quantities appear (the phase factors are unimportant since we could redefine in a suitable way the Wigner symbols). To address this issue in more details, recall from Section 2 and from [20] that the 2dimensional bistellar moves are

- The flip move, which tranforms a pair of contiguous triangles $\tau_{1}, \tau_{2}$ into another pair of triangles $\tau_{1}^{\prime}, \tau_{2}^{\prime}$ by keeping their common boundary quadrilateral fixed. Denote this move by

$$
\left[\tau_{1}, \tau_{2}\right] \longrightarrow\left[\tau_{1}^{\prime}, \tau_{2}^{\prime}\right]
$$

The algebraic identity representing this move is

$$
\begin{aligned}
& \sum_{q} \sum_{\kappa, \kappa^{\prime}}(2 q+1)(-1)^{2 q}(-1)^{-\kappa-\kappa^{\prime}}\left[\begin{array}{ccc}
p & a & q \\
\psi & \alpha & -\kappa
\end{array}\right]_{\tau_{1}}\left[\begin{array}{ccc}
q & b & r \\
\kappa & \beta & \rho
\end{array}\right]_{\tau_{2}} \\
& {\left[\begin{array}{ccc}
p & a & q \\
\psi^{\prime} & \alpha^{\prime} & -\kappa^{\prime}
\end{array}\right]_{\tau_{1}}\left[\begin{array}{ccc}
q & b & r \\
\kappa^{\prime} & \beta^{\prime} & \rho^{\prime}
\end{array}\right]_{\tau_{2}}=\sum_{c} \sum_{\gamma, \gamma^{\prime}}(2 c+1)(-1)^{2 c}(-1)^{-\gamma-\gamma^{\prime}} } \\
& {\left[\begin{array}{ccc}
a & b & c \\
\alpha & \beta & \gamma
\end{array}\right]_{\tau_{1}^{\prime}}\left[\begin{array}{ccc}
r & p & c \\
\rho & \psi & -\gamma
\end{array}\right]_{\tau_{2}^{\prime}}\left[\begin{array}{ccc}
a & b & c \\
\alpha^{\prime} & \beta^{\prime} & \gamma^{\prime}
\end{array}\right]_{\tau_{1}^{\prime}}\left[\begin{array}{ccc}
r & p & c \\
\rho^{\prime} & \psi^{\prime} & -\gamma^{\prime}
\end{array}\right]_{\tau_{2}^{\prime}} }
\end{aligned}
$$

where Latin letters denote angular momentum variables, Greek letters the first set of momentum projections and primed Greek letters the second one. A label associated with the triangles involved in the move has been added.

- The Alexander (or barycentric) move which amounts to adding one vertex in the interior of a triangle $\tau$ giving rise to three triangles $\tau_{1}^{\prime}, \tau_{2}^{\prime}, \tau_{3}^{\prime}$ bounded by the original one. We denote this operation and its inverse move by

$$
[\tau] \longleftrightarrow\left[\tau_{1}^{\prime}, \tau_{2}^{\prime}, \tau_{3}^{\prime}\right]
$$

and the corresponding identity reads

$$
\begin{aligned}
\sum_{q, r, p}(2 q+1) & (2 r+1)(2 p+1)(-1)^{2 q+2 r+2 p} \sum_{\kappa, \kappa^{\prime}} \sum_{\rho, \rho^{\prime}} \sum_{\psi, \psi^{\prime}}(-1)^{-\kappa-\kappa^{\prime}}(-1)^{-\rho-\rho^{\prime}} \\
& (-1)^{-\psi-\psi^{\prime}}\left[\begin{array}{ccc}
p & a & q \\
\psi & \alpha & -\kappa
\end{array}\right]_{\tau_{1}^{\prime}}\left[\begin{array}{ccc}
q & b & r \\
\kappa & \beta & -\rho
\end{array}\right]_{\tau_{2}^{\prime}}\left[\begin{array}{ccc}
r & c & p \\
\rho & \gamma & -\psi
\end{array}\right]_{\tau_{3}^{\prime}} \\
& {\left[\begin{array}{ccc}
p & a & q \\
\psi^{\prime} & \alpha^{\prime} & -\kappa^{\prime}
\end{array}\right]_{\tau_{1}^{\prime}}\left[\begin{array}{ccc}
q & b & r \\
\kappa^{\prime} & \beta^{\prime} & -\rho^{\prime}
\end{array}\right]_{\tau_{2}^{\prime}}\left[\begin{array}{ccc}
r & c & p \\
\rho^{\prime} & \gamma^{\prime} & -\psi^{\prime}
\end{array}\right]_{\tau_{3}^{\prime}}=} \\
& \Lambda(L)^{-1}\left[\begin{array}{ccc}
a & b & c \\
\alpha & \beta & \gamma
\end{array}\right]_{\tau}\left[\begin{array}{ccc}
a & b & c \\
\alpha^{\prime} & \beta^{\prime} & \gamma^{\prime}
\end{array}\right]_{\tau}
\end{aligned}
$$


By comparing the content of the above identities with the expression of the holographic state functional (35) we observe that

- The asymptotic value of the $6 j$ symbol corresponding to a tetrahedron of type $\sigma_{E}$ (see (22)) provides a $d$-function which decorates the edge $j$ in $\Sigma^{\text {out }}$. Thus, if we consider a pair of triangular faces sharing this edge, we cannot perform on them the flip move (37) anymore.

- From the asymptotic value (20) for the $6 j$ symbol of type $\sigma_{F}$ we get a decoration of a face in $\Sigma^{\text {out }}$. Then the barycentic move $[\tau] \rightarrow\left[\tau_{1}^{\prime}, \tau_{2}^{\prime}, \tau_{3}^{\prime}\right]$ is forbidden in such a circumstance.

- Finally, the asymptotics of the $6 j$ symbol of type $\sigma_{V}$ generates a decoration of a vertex in $\Sigma^{o u t}$. As a consequence of that, the inverse barycentric move $\left[\tau_{1}^{\prime}, \tau_{2}^{\prime}, \tau_{3}^{\prime}\right] \rightarrow$ $[\tau]$ turns out to be forbidden too.

Summing up, none of the moves represented by the identities (38) and (40) can be freely performed on the functional (35) and thus, owing to [23], the topological invariance cannot be restored in the holographic theory.

\section{Comments and remarks on the holographic theory}

We now examine in more detail some aspects of the boundary theory obtained and suggest possible lines of further investigations.

First of all we find agreement with the holographic principle in general. Indeed the boundary answer is expected to depend on the asymptotic properties of the manifold. In other words, one is examining the asymptotic behaviour of the bulk fluctuations in a region close to the boundary and according to their behaviour one may have different projections. It was guaranteed that the topological invariance of the theory would be broken by our limit as the limit basically freezes the standard triangulation as special thus breaking the symmetry between this configuration and all the other configurations that were obtainable by shelling moves.

Notice however that the theory is not as far from a topological theory as it may seem. In fact if we had included non-trivial fixed connection in the boundary theory (which indeed is necessary in the end for our path sum to have correct gluing properties) then we would have found that every edge of the dual graph on the boundary would carry a Wigner function corresponding to the connection on that edge [12, 16] and thus of the same form as the Wigner $d$ function that we find from the tetrahedra $\sigma_{E}$ in (35). In the discussion of [12] this is very similar to the squashed partition functions that are shown to be topological invariant two-dimensional path-sums. In our case the various additional factors that accompany this sum break this topological invariance, and in addition the Wigner $d(\theta)$ depend on the asymptotics of the manifold, whereas the Wigner functions that come from the boundary conditions depend upon the boundary geometry only. The dependence of $d(\theta)$ on asymptotics is very suggestive of the derivation used in [25] where the asymptotics on connections is the key to reducing a topological boundary theory to Liouville theory.

In the case of AdS/CFT this dependence on asymptotic geometry turns out to be crucial 
when evaluating the boundary correlators. But also in the original 't Hooft formulation this "near horizon" region is precisely the one in which one has interactions and ultimately correlations between ingoing and outgoing observables describing the black hole. Again the geometry of the black hole which induces violent boosts when a particle approaches the horizon, is responsible for these high energy scatterings whose phase shifts build up the hologram on the horizon.

As pointed out in [16] this asymptotic dependence turns out to be a general feature when implementing decoupling limits in the Ponzano-Regge set up. In particular the configuration considered in the previous section can be interpreted as follows: once the scale $\mathrm{R}$ is introduced one has a family of regions labelled by $\mathrm{R}$ inside the bulk which can be thought of as a sort of internal fluctuating nuclei and at the same time one is isolating the asymptotic dependence of the bulk fluctuations by progressively integrating out a region of size $\mathrm{R}$ containing tetrahedra which act as messengers of bulk information.

The boundary will behave as a screen storing bulk information depending on the value of what has been called the projection map. Note however that one can always say (independently of the value of the screen map) that the dominant contribution will be given by those tetraedra having a face on the boundary, followed by the ones which have an edge on the boundary and finally by those with only one vertex. This follows from the power law $R$ decay previously considered in the asymptotic formulae for the coupling tetraedra and gives a simple criterium to examine the spectrum of fluctuations of the boundary theory.

The bulk dependence of the boundary partition function is intimately related to the asymptotic behaviour of bulk fluctuations. One has in other words a sort of dressing of the boundary theory induced by the gravitational bulk fluctuations. This is in some sense similar to the AdS/CFT case where the source terms for the boundary theory couple a boundary operator to the asymptotic behaviour of a bulk field. In other words one might say that the bulk theory only sees, through the asymptotic behaviour of its fields, the "abstract" boundary theory and not its elementary fields.

In particular the boundary functional (35) can be associated with a fat trivalent graph (dual to the 2-dimensional triangulation) containing a pair of $3 \mathrm{jm}$ symbols for each vertex and Wigner functions connecting them. Now it is a classical result that the Teichmüller space of a Riemann surface can be combinatorially described in terms of trivalent ribbon graphs. So our boundary theory seems to have the right structure in order to construct a Teichmüller space though a precise link between our colourings and the moduli of the surface still needs to be found. Let us also point out that the Teichmüller space of the $2 \mathrm{~d}$ boundary theory should be indeed related to Einstein gravity in the three dimensional spacetime [26].

The boundary theory is then constructed by multiplying together $3 \mathrm{jm}$ symbols associated with $S U(2)$ representations. On the other hand according to standard arguments we expect to have on the boundary some kind of WZW theory and eventually Liouville theory. As a preliminary step it would then be interesting to have a dictionary for our boundary theory formulated in terms of group theory language, from a CFT perspective in a similar spirit as the dictionary proposed by Moore and Seiberg [27 between group theory and classical CFT. They show that to a compact group G there corresponds a chiral algebra and to Clebsch-Gordan coefficients correspond chiral vertex operators. Invariant tensors built up in group theory correspond to the conformal blocks of the CFT. In 
addition Racah coefficients are associated with the fusion matrix and in particular for the case of $S U(2)$ its matrix elements are nothing else than $6 j$ symbols. Functions on the group are then the physical fields of the CFT and the product of functions on the group corresponds therefore to the OPE. The average of this product represents the physical correlation functions. Therefore the product of $3 \mathrm{jm}$ symbols is related to the product of matrix elements of chiral vertex operators and according to the BPZ program it would then be possible to determine all physical quantities of the CFT. We find it intriguing that the boundary theory that is represented by our rescaled path-sum may be related by a suitable dictionary to an (as yet unspecified!) CFT.

Of course to get a precise definition of the boundary theory one should consider the dual model living in the bulk Anti-de Sitter three dimensional spacetime. The natural candidate would be the Turaev-Viro partition sum being a discretized gravity model which accomodates for the presence of a non vanishing cosmological constant. This model would also provide a well defined partition sum function being a regularized version of the Ponzano-Regge model[17, 28]. However it is not clear how a decoupling procedure of the kind proposed in Section 3 could be implemented in such a context as the quantum group spin labels are strictly bounded above and maybe another approach is necessary. A preliminary question is indeed to select the relevant representations to be summed over in the partition function of the bulk model. Now it appears that in order to implement conformal bootstrap program in LFT the algebra of conformal blocks in LFT has to be related to the algebra of irreps of a certain quantum group 229. A natural guess is then to construct a partition function in which one sums over these classes of representations [16, 30].

According to some recent analysis [29] these representations form a series of continuous irreps of $S L_{q}(2, R)$ with the deformation parameter

$$
q=\exp \left(\pi i b^{2}\right)
$$

$b \in(0,1)$ and irrational. This series has a very important property: it closes under taking tensor products of reps and this would assure the triangulation independence of the bulk model.

However the bulk model would not be automatically guaranteed to be finite since one has first to give sense to the integrals over these continuous series of representations. In other words one is faced up with the well known problem of constructing simplicial partition functions in the case in which the group is non compact. In addition, this series of representations turns out not to have a classical limit, corresponding to the Ponzano-Regge model.

As an aside remark one could consider the case of hyperbolic $2+1$ dimensional manifolds, having in mind the AdS case to get a concrete correspondence with LFT. One is then led to the notion of the so called hyperbolic knot, a knot that has a component that can be given a metric of costant negative curvature. Now the volume of the complement is a topological invariant, called the hyperbolic volume of a knot. In particular one can show that the quantum dilogarithm can lead to a generalized deformed notion of the hyperbolic volume. This seems to imply that a simplicial TQFT defined by the quantum dilogarithm can in principle be associated to $2+1$ dimensional quantum gravity. We refer to [31] and 
corresponding references for a discussion of these points.

Finally we would like to note that our example here is only three-dimensional and due to the topological nature of gravity in three dimensions is somewhat special as all degrees of freedom necessarily reside on the boundary. This model is useful however in the context of studying holography in discretized gravity as it provides an indication of how to proceed to study holography in discretized gravity in higher dimensions (for a review of higher dimensional discretized gravity see [8]). Furthermore, the eventual derivation of discretized Liouville theory using our methods would be a very interesting and non-trivial result in its own right.

\section{Acknowledgements}

G.A. would like to thank E.Martinec for a pleasant discussion during the Amsterdam Summer Workshop on String Theory. M.O'L would like to thank M. Blau and G. Thompson for various topological conversations. G.A. and M.O'L. are supported by the EEC under RTN program HPRN-CT-2000-00131. G.A. is also partially supported by MURST. M.C. and A.M. are partially supported by MURST, under PRIN grant 9901457493 (Geometry of Integrable Systems).

\section{References}

[1] G. 't Hooft, Dimensional Reduction in Quantum Gravity" in Salamfest, Trieste, 284 (1993), gr-qc/9310026.

[2] L. Susskind, The World as an Hologram, J. Math. Phys. 366377 (1995), hepth/9409089.

[3] T. Banks, W. Fischler, S. Shenker, L. Susskind, M Theory As A Matrix Model: A ConjecturePhys. Rev. D55 5112 (1997), hep-th/9610043.

[4] J. Maldacena, The large N limit of superconformal field theories and supergravity, Adv. Theor. Math. Phys. 2, 231 (1998), hep-th/9711200.

[5] O. Aharony, S.S. Gubser, J. Maldacena, H. Ooguri and Y. Oz, Large N Field Theories, String Theory and Gravity, Phys.Rept. 323 183-386 (2000), hep-th/9905111.

[6] G. 't Hooft, The scattering matrix approach for the quantum Black Hole- an O verview, Int. J. Mod. Phys. A11 4623-4688 (1996), gr-qc/9607022 and references therein.

[7] G.'t Hooft, Transplanckian particles and the quantization of time, Class. Quant. Grav. 16 395-405 (1999), gr-qc/9805079.

[8] T. Regge and R. M. Williams, Discrete structures in gravity, J.Math.Phys. 41 39643984 (2000), gr-qc/0012035.

\footnotetext{
${ }^{6}$ We also point out that the same author has been relating the quantum hyperbolic invariant of knots to the quantum theory of Teichmüller spaces of punctured surfaces [32].
} 
[9] N. Kawamoto, H.B. Nielsen and N.Sato, Lattice Chern-Simons Gravity via PonzanoRegge Model, Nucl. Phys. B555 (1999), 629, hep-th/9902165.

[10] P. Hořava, M-theory as a holographic field theory, Phys. Rev. D59: 046004 (1999), hep-th/9712130.

[11] G. Ponzano, T. Regge, Semiclassical Limit of Racah Coefficients, in Spectroscopic and Group theoretical Methods in Phyisics p.1-58, ed. F. Bloch, (North-Holland, 1968).

[12] H. Ooguri, Partition functions and topology-changing amplitudes in the three dimensional lattice gravity of Ponzano-Regge, Nucl. Phys. B382, 276-304, (1992).

[13] G. Carbone, M. Carfora and A. Marzuoli, Wigner symbols and combinatorial invariants of three-manifolds with boundary, Comm. Math. Phys. 212, 571-590 (2000).

[14] G. Carbone, M. Carfora and A. Marzuoli, Hierarchies of invariant spin models, Nucl. Phys. B595 [PM], 654-688 (2001), gr-qc/0008011.

[15] U. Pachner, P.L. homeomorphic manifolds are equivalent by elementary shellings, Europ. J. Combinatorics 12, 129-145 (1991).

[16] M. O'Loughlin, Boundary actions in Ponzano-Regge discretization, quantum groups and $A d S_{3}$, gr-qc/0002092.

[17] V. Turaev and O.Y. Viro, State sum invariants of 3-manifolds and quantum $6 j$ symbols, Topology 31, 865-902,(1992).

[18] T. Regge, General Relativity without coordinates, Nuovo Cimento 19, 558-571, (1961).

[19] J. B. Hartle and R. Sorkin, Boundary Terms in the Action for the Regge Calculus, Gen. Rel. Grav. 13, 541-549 (1981).

[20] G. Carbone, M. Carfora and A. Marzuoli, Invariants of spin networks with boundary in quantum gravity and TQFTs, gr-qc/9905076.

[21] S. Carlip, Quantum Gravity in 2+1 Dimensions, Cambridge University Press (1998).

[22] J. Ambjorn, B. Durhuus and T. Jonsson, Quantum Geometry, Cambridge University Press (1997).

[23] U. Pachner, Ein Henkeltheorem für geschlossene semilineare Mannigfaltigkeiten, Result. Math. 12, 386-394 (1987).

[24] D.A. Varshalovich, A.N. Moskalev and V.K. Khersonskii, Quantum Theory of Angular Momentum, World Scientific, Singapore (1988).

[25] J.D. Brown and M. Henneaux, Central charges in the canonical realization of asymptotic symmetries: an example from three-dimensional gravity, Comm.Math.Phys.104(1986), 207. O. Coussaert, M. Henneaux and P. van Driel, The asymptotic dynamics of three-dimensional Einstein gravity with a negative cosmological constant, gr-qc/9506019. 
[26] H. Verlinde, Conformal field theory, two dimensional quantum gravity and quantization of Teichmüller space, Nucl. Phys. B337 652 (1990).

[27] G. Moore, N. Seiberg, Classical and Quantum Conformal Field Theory, Commun. Math. Phys. 123, 177-254, (1989).

[28] S. Mizoguchi and T. Tada, 3-Dimensional Gravity and the Turaev-Viro Invariant, Progr. Theor. Phys. Suppl. 110, 207-227, (1992).

[29] J. Teschner, Liouville Theory Revisited, hep-th/0104158 and references therein.

[30] K. Krasnov, 3D Gravity, Point Particles and Liouville Theory, Class. Quant. Grav. 18: 1291-1304 (2001). hep-th/0008253.

[31] R. M. Kashaev, The hyperbolic volume of knots from quantum dilogarithm, Lett. Math. Phys. 39: 269-275 (1997), q-alg/9601025 and references therein.

[32] R. M. Kashaev, Quantization of Teichmüller spaces and the quantum dilogarithm, q-alg/9705021. 